\title{
Human nutrition, environment, and health
}

\author{
Jim Kaput ${ }^{1}$ Martin Kussmann ${ }^{1}$ - Marijana Radonjic ${ }^{2}$ - Fabio Virgili ${ }^{3}$. \\ Giuditta Perozzi ${ }^{3}$
}

Published online: 26 August 2015

(c) Springer-Verlag Berlin Heidelberg 2015

Improving health of individuals who consume too many or too few calories or inadequate levels of micro- and macronutrients remains a major challenge for today's world. Providing fresh and healthy manufactured foods in a sustainable manner for future generations is predicted to become more difficult because the earth's population is expanding and aging, chronic diseases are surging due to obesity, and local and global environments are changing at accelerating rates. Nutrition research needs to play a major role in providing evidence-based recommendations to policy makers, the agricultural community, and the general public to meet the challenges expected in the coming decades.

The nutrition research community has contributed significantly to the virtual elimination of single-nutrient deficiencies that cause, for example, beriberi, rickets, pellagra, and scurvy. The success of these interventions contributed to the belief that single or a few micronutrients might alleviate or eliminate growth and cognitive impairments or prevent the development of other chronic conditions such as obesity and diabetes. In addition, many nutrition studies have too few participants, measured and analyzed a limited sets of molecular readouts and

This Editorial is the introduction to the Topical Collection on Systems Health and Nutrition, guest edited by Jim Kaput, Martin Kussmann, and Marijana Radonjic. The Guest Editors wrote this Editorial, along with the founding editors of Genes and Nutrition, Fabio Virgili and Giuditta Perozzi.

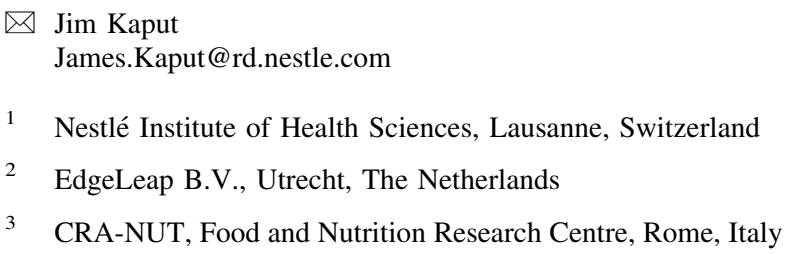

phenotypic endpoints, lacked genetic analysis, and were geographically specific with insufficient analysis of environmental factors that may influence physiology. Typically, studies were conducted independently of other interventions with different designs or endpoints, hampering generation of knowledge for improving or maintaining health across different genetic or cultural populations and for consumers and patients as individuals. Witness the epidemic in diabetes, obesity, and other lifestyle-related diseases while almost 1 billion people lack quality nutrition for reaching their full physical and cognitive development.

The limitations of 20th century nutrition research were recognized and publicized in the early 2000s (Kaput et al. 2005). The decade of nutrition research since has seen the development and use of high-throughput phenotyping methods, improved study designs, and the integration of genomic data. These advances now allow a means to unravel food-health interactions at multiple levels of biological complexity as well as the ability to design nutritional strategies to maintain health and delay or prevent onset of lifestyle diseases. The result of these developments led to refinements in thinking and strategies best summarized as systems nutrition (van Ommen 2004; Kaput et al. 2014). This Topical Collection is another touchstone in the development of concepts and applications of this new research strategy to nutrition and health research. The editors and publisher (Springer) of Genes and Nutrition decided to focus on this topic as a result of three related events: (i) a series of workshops on essential nutrients (see Kaput et al. 2014) including the 4th on systems analysis of genetic data that was held in September 2014 at the Microsoft Research-University of Trento Institute for Computation and Systems Biology (COSBI.eu), (ii) the Bill and Melinda Gates Grand Challenge Meeting (Beijing, October 2015) whose focus is on nutrition, which partnered 
with (iii) the first Keystone Symposium that focuses on Human Nutrition, Environment and Health (Beijing, October 2015). The combination of this topical collection and meetings hosted by groups beyond the nutrition community herald the recognition that systems nutrition and health research is an established discipline in the scientific community.

The Systems Nutrition Topical Collection highlights reviews, examples of systems nutrition research, and expansion of systems analysis from within-body measurements to the entire nutrient chain from soil to fork (http:// tinyurl.com/onosror).

- Systems nutrition research inherently acknowledges that the environment influences the biological system. Measuring environmental variables has generally been limited to diet and occasionally selected other lifestyle variables such as activity. Nutritional quality is greatly influenced by soils and climates where food is grown and how and where it is transported and stored. Food accessibility and price also differentially affect nutrient intake across the socioeconomic spectrum. In addition, the food production system is now global, and each of these factors separately and interactively influences our local food environment. The growing awareness that climate change and population growth is already challenging our ability to produce enough nutritious foods now and over the next decades has uncovered the need to connect basic nutrition research to the web of the food supply system (Kaput et al. 2015).

- The conceptual and methodological framework of systems nutrition is reviewed in relation to micronutrient requirements for different genetic backgrounds (Monteiro et al. 2015). The limited translatability of population-level data to individuals emphasizes the importance of experimental designs focusing on n-of-1 as a complement to the typical case/control experimental designs. Different responses to micro- and macro-nutrients result from the interactions of individual genetic makeups being influenced differently by the environment-individuals may, and usually do, respond differently to the same nutritional intervention.

- Systems thinking and approaches must be applied to the continuum of health to disease. Zhao et al. describe the applications of omics integration to type 2 diabetes research (Zhao et al. 2015). Chemicals in the diet alter transcriptomic, proteomic, metabolomic, epigenetic, and microbial profiles as has been shown in human and animal studies. The key challenge of systems approaches is the integration of the data to produce actionable results for both healthy and diseased individuals.
- Biological processes are dynamic and therefore the underlying networks change depending on input into the system. Derous et al. employed network-based modeling as a powerful platform for integration and mining of data and knowledge to comprehensively characterize adaptation of mouse white adipose tissue to high-fat diet, spanning from global aspects to mechanistic details (Derous et al. 2015). A threelayered interaction network model comprising enriched biological processes was built and functionally analyzed, linked to transcriptional regulators within the network, and the network was associated with changes in physiological parameters. The resulting knowledge base provided novel mechanistic insights in appetite control, high fat-induced pathology, and clues to an intervention strategy to effectively deal with excess dietary fat.

- Although the concept of systems network analysis is still emerging, existing data sets in the public domain can be exploited to generate new insights and results. Kutmon et al. uses a publicly available data set of changes in transcript and microRNA abundances in prostate cancer cells treated with 1,25-dihydroxyvitamin D3 (Kutmon et al. 2015). PathVisio and Cytoscape network tools identified cell cycle and cancer-specific pathways altered by this vitamin.

- In 1948, the WHO defined health as a condition of complete physical, mental, and social well-being and not the mere absence of disease. This definition framed nutritional and biomedical research toward an unachievable goal of perfection across all life stages. A newer, more nuanced definition was proposed in 2011: health is the ability to physical, cognitively, and socially adapt to continuing changes in the environment (Huber et al. 2011), a concept that was extended to metabolic flexibility (van Ommen et al. 2014). Stroeve et al. describe the concepts of metabolic flexibility and the different experimental nutritional approaches that are used to challenge homeostasis (Stroeve et al. 2015). The majority of these challenges are acute challenges of carbohydrate, protein, or lipid loads that elicit a strong metabolic response in the hours after ingestion. The responses of different biological processes to such macronutrient loads are monitored at the level of specific metabolites, proteins, and other circulating molecules. Individuals differ in how they respond to these metabolic challenges (Krug et al. 2012). These new approaches are crucial to the development of nutrition knowledge, but also new combinations of foods that may emerge as the human population faces changes in the food system caused by climate change and population growth. 
- This systems nutrition topical collection is a rolling publication-we will be adding solicited and unsolicited contributions for electronic publication over the next 2 years. Our goal is to stimulate research in systems nutrition and its translatability to improve personal and public health.

The published systems nutrition (and more so, systems biology) studies identified key gaps to improve translatability of the results: (i) the challenge of integrating different data types in computational models, and (ii) the necessity of capturing a wider range of quantitative measures of environment and physiology that alter health or disease processes, even if these data are not directly used in analyses (metadata or context information), and (iii) effective integration of data and metadata with prior knowledge of health-relevant interactions to facilitate interpretation, novel discovery, hypothesis generation, and validation of results (Kaput et al. 2014; Kelder et al. 2015). Physiological processes will be influenced, directly or indirectly, by access to healthcare and other social systems, sanitation, and clean water, by the change of nutritional quality of food from soil to table, and by food availability and cost. That is, the context of the individual's setting is crucial for interpreting experimental results of nutritional (and other) interventions. Quantitative assessments of these factors have not been adequately captured in current experimental designs or accounted for in data analyses. Nutrition research requires a more complete description of environmental and experimental variables for more comprehensive interpretation of complex, high-dimensional data. Optimal nutrition is required to reach peak physical and cognitive development and performance, and cannot be achieved by addition of single micronutrients to poor quality diets. Hence, the next step in improving personal and public health, by nutrition and lifestyle, is to understand the interactions between the essential nutrients and how these interactions are affected by human and microbial (epi) genetic diversity. In doing so, appropriate metabolic improvements and optimization can be achieved across all organ systems (Ames 2003), that is, a holistic rather than symptom centered approach to health. Ideally, this new knowledge will be available to individuals but realistically, food and lifestyle recommendations will first be targeted to groups of individuals with similar metabolic needs and response.

We also suggest that a key element of systems nutrition research is the ability to aggregate data from diverse regions and populations throughout the world since genetic makeup alters response to food and drugs. Expanding nutrition and health research capabilities in the global community is especially important as climate change alters local environmental conditions that influence food and agricultural production. A necessary step toward this goal will be to build research capacity and infrastructure in lowand middle-income countries (LMIC) to enhance development of translational research programs (McCabe-Sellers et al. 2008) for more rapid implementation and application of knowledge. In most cases, building such capability, capacity, and infrastructure requires the involvement of local governments and agencies, a process consistent not only with the goal of knowledge and technology transfer from North to South (Seguin et al. 2008), but also the stimulation of economic development through research activities [see www.cohred.org and (Tindana et al. 2007; Singh and Daar 2008)]. The Human Variome Project, which seeks to understand the genetic basis of human disease (Oetting 2007; Kaput et al. 2009; Cotton and Macrae 2010), has initiated these types of capacity building projects by establishing strategies to collect data from clinics and research laboratories in LMIC (Al Aama et al. 2011).

The Human Nutrition, Environment and Health conference is another step in developing a global community for systems nutrition research. The Keystone Symposium, the BMGF, and the U.S. NIH contributed financial resources to include researchers from LMIC to speak at or participate in the conference. A pre-conference workshop provided additional information on the concepts, terminology, and strategies of network biology to attendees from these countries. These efforts to include researchers from around the world in this new science build on previous systems nutrition conferences and workshops (e.g., Kaput et al. 2014) supported by the Nestle Institute of Health Sciences (nestleinstitutehealthsciences.com), NuGO (NUGO.org), COSBI (COSBI.eu), Wageningen University through its graduate school VLAG (vlaggraduateschool.nl), DSM (DSM.com), and the BMGF (gatesfoundation.org).

Systems-level thinking and high-throughput omics methods have emerged over the past decade and are now at an actionable stage. Curated databases are being developed in the nutrition field (van Ommen et al. 2010) and computational methods are maturing to approach multi-level, multi-source data integration and analysis, including dynamics. Novel standards for data capturing and sharing are being set: generated data and relations need to be documented as traceable, transparent, annotated and accessible resources, ideally in a computer readable format ready for efficient mining and cross-validation by the broader scientific community. The health benefits of an enhanced understanding of the effects of both nutritional deprivation and supplementation, at a systems level, will contribute to improved outcomes such as prevention of stunting, improved growth and physical development, reduced susceptibility to infectious diseases, improved 
capacity to recover from infections, and improved cognitive development.

\section{Compliance with ethical standards}

Disclosure JK and MK are employed by the for-profit Nestlé Institute of Health Sciences. MR is founder and CEO of EdgeLeap B.V.

\section{References}

Al Aama J, Smith TD, Lo A et al (2011) Initiating a Human Variome Project country node. Hum Mutat 32:501-506. doi:10.1002/ humu. 21463

Ames BN (2003) The metabolic tune-up: metabolic harmony and disease prevention. J Nutr 133:1544-1548

Cotton RG, Macrae FA (2010) Reducing the burden of inherited disease: the Human Variome Project. Med J Aust 192:628-629

Derous D, Kelder T, van Schothorst EM et al (2015) Network-based integration of molecular and physiological data elucidates regulatory mechanisms underlying adaptation to high-fat diet. Genes Nutr 10:22. doi:10.1007/s12263-015-0470-6

Huber M, Knottnerus JA, Green L et al (2011) How should we define health? BMJ 343:d4163. doi:10.1136/bmj.d4163

Kaput J, Ordovas JM, Ferguson L et al (2005) The case for strategic international alliances to harness nutritional genomics for public and personal health. Br J Nutr 94:623-632

Kaput J, Cotton RG, Hardman L et al (2009) Planning the Human Variome Project: the Spain report. Hum Mutat 30:496-510. doi:10.1002/humu.20972

Kaput J, van Ommen B, Kremer B et al (2014) Consensus statement-understanding health and malnutrition through a systems approach: the ENOUGH program for early life. Genes Nutr 9:378. doi:10.1007/s12263-013-0378-y

Kaput J, Kussmann M, Mendoza Y et al (2015) Enabling nutrient security and sustainability through systems research. Genes Nutr 10:12. doi:10.1007/s12263-015-0462-6

Kelder T, Summer G, Caspers M et al (2015) White adipose tissue reference network: a knowledge resource for exploring healthrelevant relations. Genes Nutr 10:439. doi:10.1007/s12263-0140439-x
Krug S, Kastenmüller G, Stückler F et al (2012) The dynamic range of the human metabolome revealed by challenges. FASEB J 26:2607-2611. doi:10.1096/fj.11-198093

Kutmon M, Coort SL, de Nooijer K et al (2015) Integrative networkbased analysis of mRNA and microRNA expression in 1,25dihydroxyvitamin D3-treated cancer cells. Genes Nutr 10:35. doi:10.1007/s12263-015-0484-0

McCabe-Sellers B, Lovera D, Nuss H et al (2008) Personalizing nutrigenomics research through community based participatory research and omics technologies. OMICS 12:263-272. doi:10. 1089/omi.2008.0041

Monteiro JP, Kussmann M, Kaput J (2015) The genomics of micronutrient requirements. Genes Nutr 10:19. doi:10.1007/ s12263-015-0466-2

Oetting WS (2007) The 2006 Human Genome Variation Society scientific meeting. Hum Mutat 28:517-521. doi:10.1002/humu. 20489

Seguin B, Hardy BJ, Singer PA, Daar AS (2008) Genomic medicine and developing countries: creating a room of their own. Nat Rev Genet 9:487-493. doi:10.1038/nrg2379

Singh JA, Daar AS (2008) The 20-year African biotech plan. Nat Biotechnol 26:272-274. doi:10.1038/nbt0308-272

Stroeve J, van Wietmarschen H, Kremer B et al (2015) Phenotypic flexibility as a measure of health: the optimal nutritional stress response test. Genes Nutr 10:13. doi:10.1007/s12263-015-0459-

Tindana PO, Singh JA, Tracy CS et al (2007) Grand challenges in global health: community engagement in research in developing countries. PLoS Med 4:e273. doi:10.1371/journal.pmed.0040273

Van Ommen B (2004) Nutrigenomics: exploiting systems biology in the nutrition and health arenas. Nutrition 20:4-8

Van Ommen B, Bouwman J, Dragsted LO et al (2010) Challenges of molecular nutrition research 6: the nutritional phenotype database to store, share and evaluate nutritional systems biology studies. Genes Nutr 5:189-203. doi:10.1007/s12263-010-0167-9

Van Ommen B, van der Greef J, Ordovas JM, Daniel H (2014) Phenotypic flexibility as key factor in the human nutrition and health relationship. Genes Nutr 9:423. doi:10.1007/s12263-0140423-5

Zhao Y, Barrere-Cain RE, Yang X (2015) Nutritional systems biology of type 2 diabetes. Genes Nutr 10:31. doi:10.1007/s12263-015$0481-3$ 